\title{
IMAGING BEYOND THE PINHOLE CAMERA
}

\author{
Co-chairs: \\ Kostas Daniilidis (USA), Reinhard Klette (New Zealand), \\ and Ales Leonardis (Slovenia)
}

Seminar at Dagstuhl, 13 - 18 June 2004

The world's first photograph was taken by Joseph Nicphore Niépce (1775-1833) in 1826 on his country estate near Chalon-sur-Sane, France. The photo shows parts of farm buildings and some sky. Exposure time was eight hours. Niépce used a pinhole camera, known as camera obscura, and utilized pewter plates as the support medium for the photographic process. The camera obscura, the basic projection model of pinhole cameras, was first reported by the Chinese philosopher Mo-Ti (5th century BC): light rays passing through a pinhole into a darkened room create an upside-down image of the outside world.

Cameras used since Niépce are basically following the pinhole camera principle. The quality of projected images improved due to progress in optical lenses and silver-based film, the latter one replaced today by digital technologies. Pinhole-type cameras are still the dominating brands, and also used in computer vision for understanding 3D scenes based on captured images or videos.

However, different applications have pushed for designing alternative architectures of cameras. For example, in photogrammetry cameras are installed in planes or satellites, and a continues stream of image data can also be created by capturing images just line by line, one line at a time. As a second example, robots require to understand a scenery in full $360^{\circ}$ to be able to react on obstacles or events; a camera looking upward into a parabolic or hyperbolic mirror allows this type of omnidirectional viewing. The development of alternative camera architectures also requires to understand related projective geometries for the purpose of camera calibration, binocular stereo, or static or dynamic scene understanding.

This abstract collection reports about contributions given at a workshop at the international computer science center in Dagstuhl (Germany) addressing basics and applications of alternative camera technologies, in particular in the context of computer vision, computer graphics, visualisation centers, camera producers, or application areas such remote sensing, surveillance, ambient intelligence, satellite or super-high resolution imaging. Examples of subjects are geometry and image processing on plenoptic modalities, multiperspective image acquisition, panoramic imaging, plenoptic sampling and editing, new camera technologies and related theoretical issues.

This abstract collection is structured into five parts on (1) sensor geometry for different camera architectures, also adressing calibration, (2) applications of non-pinhole cameras for analyzing motion, (3) mapping of 3D scenes into 3D models, (4) navigation of robots using new camera technologies, and (5) on specialized aspects of new sensors and other modalities.

New results and specific research strategies have been discussed at this seminar to approach this highly complex field. The seminar intention was to discuss theoretical fundamentals related to those issues and to specify open problems and major directions of further development in the field of new camera technologies related to computer vision, computer graphics and related applications. The seminar schedule was characterised by flexibility, working groups, and sufficient time for focused discussions.

The participants of this seminar enjoyed the atmosphere and the services at Dagstuhl very much. The quality of this center is unique.

There will be an edited volume of seminar papers (within the Kluwer series) with an expected publication date in early 2006 .

Kostas Daniilidis,

Reinhard Klette, and

Ales Leonardis 


\title{
Part I - SENSOR GEOMETRY
}

\section{GEOMETRY OF A CLASS OF CATADIOPRIC SYSTEMS}

\author{
A. Torii , A. Sugimoto, T. Sakai, and A. Imiya (Japan)
}

Images observed by a catadioptric system with a quadric mirror are considered as images on a quadric surface which is determined by a mirror of the system. In this paper, we propose a unified theory for the transformation from images observed by catadioptric systems to images on a sphere. Images on a sphere are functions on a Riemannian manifold with the positive constant curvature. Mathematically, spherical images have similar analytical and geometrical properties with images on a plane. This mathematical property leads to the conclusion that spherical image analysis provides a unified approach for the analysis of images observed through a catadioptric system with a quadric mirror. Therefore, the transformation of images observed by the systems with a quadric mirror to spherical images is a fundamental tool for image understanding of omnidirectional images. We show that the transformation of omnidirectional images to spherical images is mathematically a point-to-point transformation among quadric surfaces. This geometrical property comes from the fact that the intersection of a double cone in a four-dimensional Euclidean space and a three-dimensional linear manifold yields a surface of revolution employed as a mirror for the catadioptric imaging system with a quadric mirror.

\section{UNIFYING IMAGE PLANE LIFTINGS FOR CENTRAL CATADIOPTRIC AND DIOPTRIC CAMERAS}

\section{J.P. Barreto (Portugal) and K. Daniilidis (USA)}

In this paper, we study projection systems with a single viewpoint, including combinations of mirrors and lenses (catadioptric) as well as just lenses with or without radial distortion (dioptric systems). Firstly, we extend a well-known unifying model for catadioptric systems to incorporate a class of dioptric systems with radial distortion. Secondly, we provide a new representation for the image planes of central systems. This representation is the lifting through a Veronese map of the original image plane to the $5 \mathrm{D}$ projective space. We study how a collineation in the original image plane can be transferred to a collineation in the lifted space and we find that the locus of the lifted points which correspond to projections of world lines is a plane in parabolic catadioptric systems and a hyperplane in case of radial lens distortion.

\section{GEOMETRIC CONSTRUCTION OF THE CAUSTIC SURFACE OF CATADIOPTRIC NON CENTRAL SENSORS}

\section{S. Ieng and R. Benosman (France)}

Most of the catadioptric cameras rely on the single viewpoint constraint that is hardly fulfilled. There exists many works on non single viewpoint catadioptric sensors satisfying specific resolutions. In such configur ations, the computation of the caustic curve becomes essential. Existing solutions are unfortunately too specific to a class of curve s and need heavy computation load. This paper presents a flexible geometric construction of the caustic curve of a catadioptric sensor. I ts extension to the 3D case is possible if some geometric constraints are satisfied. This introduces the necessity of calibratio $n$ that will be briefly exposed. Tests and experimental results illustrate the possibilities of the method.

\section{CALIBRATION OF LINE-BASED PANORAMIC CAMERAS}

\author{
F. Huang, S.-K. Wei (Taiwan), and R. Klette (New Zealand)
}

The chapter studies the calibration of four parameters of a rotating CCD line sensor, which are the effective focal length and the principal row (which are part of the intrinsic calibration), and the off-axis distance and 
the principal angle (which are part of the extrinsic c alibration). It is shown that this calibration problem can be solved by considering two independent subtasks, first the calibration of both intrinsic parameters, and then of both extrinsic parameters. The chapter introduces and discusses different methods for the calibration of these four parameters. Results are compared based on experiments using a super-high resolution line-based panoramic camera. It turns out that the second subtask is solved best if a straight-segment based approach is used, compared to point-based or correspondence-based calibration methods; these approaches are already known for traditional (planar) pinhole cameras, and this chapter discusses their use for calibrating panoramic cameras.

\title{
MULTIRESOLUTION ON THE SPHERE
}

\author{
M. Conrad (Germany)
}

The management of large sets of meteorological, imaging or geophysical data collected over the earth or other spheres is a challenging task. For synthesizing and analyzing such data a multiscale approach seems to be particularly adequate. The aim of this work is to summarize background definitions and some known approximation methods useful for studying multiscale approximations on the sphere. In contrast to the situation for $\mathrm{L}^{2}\left(R^{1}\right)$ or $\mathrm{L}^{2}\left(R^{m}\right)$ the construction of suitable multiscale bases for the sphere is more complicated. Let us only mention here that apart from the five regular polytopes there are no equidistant point systems on the sphere. Hence, wavelet spaces cannot be simply generated by equidistant translates of one generating function. However, a lot of different approaches try to overcome these difficulties. A quite simple technique for constructing biorthogonal wavelets on the sphere based on the lifting scheme. Another construction of spherical wavelets based on tensor products of polynomial splines and trigonometric splines. The main ingredient in our constructions turn out to be the well-known spherical harmonics. These homogeneous and harmonic polynomials restricted to the sphere play the same fundamental role as the sine and cosine frequencies for periodic functions or e.g. Legendre polynomials for the functions on the interval $[-1,1]$. In these univariate settings a lot of results concerning multiscale decompositions were published in the last years. Here we follow these ideas and relate all our constructions to these basic blocks of the underlying orthonormal system of spherical harmonics. However, let us also stress the fact that polynomials cannot give optimal localization on the sphere. This behavior can be studied in terms of uncertainty inequalities in a more quantitative way. In our paper we summarize some corresponding approximation procedures. Two different examples will be studied. A polynomial approach can be used very efficiently by applying the FFT and related fast algorithms. A second class of examples is based on spherical basis functions. In this case scattered data approximation is possible.

\section{A POSITIVE QUADRATURE RULE ON THE SPHERE}

\section{J. Prestin (Germany) and D. Roşca (Romania)}

Recently, N. Laín Fernández obtained a fundamental system of points on the two-dimensional sphere which can be used to generate a polynomial multiresolution on the sphere. In this talk we construct out of these points interpolatory quadrature rules. The weights of these rules can be computed explicitely. Of particular interest are the cases where one obtains positive weights which can be described explicitely. Furthermore, we discuss the growth of these weights. Finally, we study the possibility to obtain spherical designs of this particular interpolatory kind. It turns out that only for some small number of points such Chebyshev quadrature is possible. The particular point systems will be described explicitely. 


\title{
Part II - MOTION
}

\author{
ON CALIBRATION, STRUCTUR FROM MOTION \\ AND MULTI-VIEW GEOMETRY FOR GENERIC CAMERA MODEL
}

\author{
P. Sturm (France), S. Ramalingam, and S. Lodha (USA)
}

We consider calibration and structure from motion tasks for a previously introduced, highly general imaging model, where cameras are modeled as possibly unconstrained sets of projection rays. This allows to describe most existing camera types (at least for those operating in the visible domain), including pinhole cameras, sensors with radial or more general distortions, catadioptric cameras (central or non-central), etc. Generic algorithms for calibration and structure from motion tasks (pose and motion estimation and 3D point triangulation) are outlined. The foundation for a multi-view geometry of non-central cameras is given, leading to the formulation of multi-view matching tensors, analogous to the fundamental matrices, trifocal and quadrifocal tensors of perspective cameras. Besides this, we also introduce a natural hierarchy of camera models: the most general model has unconstrained projection rays whereas the most constrained model dealt with here is the central model, where all rays pass through a single point.

\section{MOTION ESTIMATION \\ WITH ESSENTIAL AND GENERALIZED ESSENTIAL MATRICES}

\author{
R. Molana and C. Geyer (USA)
}

Recent advances with camera clusters, mosaics, and catadioptric systems led to the notion of generalized images and general cameras, considered as the rigid set of viewing rays of an imaging system, known also as the domain of the plenoptic function. In this paper, we study the recovery of rigid 3D camera motion from ray correspondences in both cases when all rays intersect (central) or do not intersect (non-central) at a single viewpoint. We characterize the manifold associated with the central essential matrices and we show that the non-central essential matrices are permutations of SE(3). Based on such a group-theoretic parameterization, we propose a non-linear minimization on the central and non-central essential manifold, respectively. The main contribution of this paper is a unifying characterization of two-view constraints in camera systems and a computational procedure based on this framework. Current results include simulations verifying priorly known facts for the central case and showing the sensitivity in the transition from central to non-central systems.

\section{CORRESPONDENCELESS VISUAL NAVIGATION UNDER CONSTRAINED MOTION}

\section{A. Makadia and K. Daniilidis (USA)}

Visual navigation techniques traditionally use feature correspondences to estimate motion in the presence of large camera motions. The availability of black-box feature tracking software makes the utilization of correspondences appealing when designing motion estimation algorithms. However, such algorithms break down when the feature matching becomes unreliable. To address this issue, we introduce a novel approach for estimating camera motions in a correspondenceless framework. This model can be easily adapted to many constrained motion problems, and we will show examples of pure camera rotations, pure translations, and planar motions. The objective is to efficiently compute a global correlation grid which measures the relative lik elihood of each camera motion, and in each of our three examples we show how this correlation grid can be quickly estimated by using generalized Fourier tran sforms. 


\title{
SEGMENTATION OF DYNAMIC SCENES \\ TAKEN BY A MOVING CENTRAL PANORAMIC CAMERA
}

\author{
R. Vidal (USA)
}

We present an algebraic geometric solution to the problem of segmenting an unknown number of rigid-body motions from optical flow measurements taken by a moving central panoramic camera. We first show that the central panoramic optical flow generated by a rigidly moving object lives in a complex six-dimensional subspace of a high-dimensional linear space, hence motion segmentation is equivalent to segmenting data living in multiple complex subspaces. We solve this problem in closed form using complex Generalized PCA. Our approach involves projecting the optical flow measurements onto an seven-dimensional subspace, fitting a complex polynomial to the projected data, and differentiating this polynomial to obtain the the motion of each object relative to the camera and the segmentation of the image measurements. Unlike previous work for affine cameras, our method does not restrict the motion of the objects to be full-dimensional or fully independent. Instead, our approach deals gracefully with all the spectrum of possible motions: from low-dimensional and partially dependent to fulldimensional and fully independent. We test our algorithm on real sequences with degenerate and nondegenerate motions, achieving a misclassification error of less than 5\%. For a sequence with two mobile robots, we also compare the estimates of our algorithm with GPS measurements gathered by the mobile robots.

\section{OPTICAL FLOW COMPUTATION ON OMNIDIRECTIONAL IMAGES}

A. Imiya, A. Torii , and H. Sugaya (Japan)

This paper focuses on variational image analysis on Riemannian manifolds. Since a sphere is a closed Riemannian manifold with the positive constant curvature and no holes, the sphere has similar geometrical properties with a plane, whose curvature is zero. Images observed through a catadioptric system with a conic mirror is transformed to images on the sphere. As an application of image analysis on Riemannian manifolds, we develop an accurate algorithm for the computation of optical flow of omni-directional images. The spherical motion field on the spherical retina has some advantages for ego-motion estimation of autonomous mobile observer. Our method provides a framework for motion field analysis on the spherical retina, since views observed by a quadric-mirror-based catadioptric system are transformed to views on the spherical and semispherical retinas. 


\title{
Part III - MAPPING
}

\section{ENVIRONMENTAL MAP GENERATION FROM OPTICAL FLOW FOR ROBOT NAVIGATION}

\author{
N. Ohnishi and A. Imiya (Japan)
}

In this paper, we aim to develop an algorithm for the construction of an environmental map using optical flow observed through a vision system mounted on an autonomous mobile robot. Our algorithm first detects an area corresponding to the planar area from a sequence of images by employing optical flow, and second, backprojects the planar area to the environment for the construction of an environmental map. Moreover, our algorithm estimates ego-motion of the mobile robot using projected optical flow. The planar area detection by our method does not require any physical assumptions concerning the robot motion or any camera calibration. Therefore, our algorithm allows obstacles to be detected without using any models in the database or any heuristics rule. We show the results of the construction of an environmental map using the mobile robot.

\section{MOBILE PANORAMIC MAPPING USING CCD-LINE CAMERA AND LASER SCANNER WITH INTEGRATED POSITION AND ORIENTATION SYSTEM}

\author{
R. Reulke, A. Wehr, and D. Griesbach (Germany)
}

The fusion of panoramic camera data with laser scanner data is a new approach and allows the combination of high-resolution image and depth data. Application areas are city modelling, virtual reality and documentation of the cultural heritage. Panoramic recording of image data is realized by a CCD-line, which is precisely rotated around the projection centre. In the case of other possible movements, the actual position of the projection centre and the view direction has to be measured. Linear moving panoramas e.g. along a wall are an interesting extension of such rotational panoramas. Here, the instantaneous position and orientation determination can be realized with an integrated navigation system comprising differential GPS and an inertial measurement unit. This paper investigates the combination of a panoramic camera and a laser scanner with a navigation system for indoor and outdoor applications. First, laboratory experiments it are reported, which were carried out to obtain valid parameters about the surveying accuracy achievable with both sensors panoramic camera and laser scanner respectively. Thereafter, outdoor surveying results using a position and orientation system as navigation sensor are presented and discussed.

\section{MULTI-SENSOR PANORAMA FUSION AND VISUALIZATION}

\author{
K. Scheibe (Germany) and R. Klette (New Zealand)
}

The paper describes a general approach for scanning and visualizing panoramic $\left(360^{\circ}\right)$ indoor scenes. It combines range data acquired by a laser range finder with color pictures acquired by a rotating CCD line camera. The paper defines coordinate systems of both sensors, specifies the fusion of range and color data acquired by both sensors, and reports about different alternatives for visualizing the generated 3D data set. Compared to earlier publications, the recent approach also utilizes an improved method for calculating the spatial (geometric) correspondence between laser diode of the laser range finder and the focal point of the rotating CCD line camera. Calibration is also a subject in this paper. A least-square minimization based approach is proposed for the rotating CCD line camera.

\section{MULTI-PERSPECTIVE MOSAICS FOR INSPECTION AND VISUALIZATION}

A. Koschan, J.-C. Ng, and M. Abidi (USA)

In this chapter, we address the topic of building multi-perspective mosaics of infra-red and color video 
data acquired by moving cameras under the constraints of small and large motion parallaxes. We distinguish between techniques for image sequences with small motion parallaxes and techniques for image sequences with large motion parallaxes and we describe techniques for building the mosaics for the purpose of under vehicle inspection and visualization of roadside sequences. For the under vehicle sequences, the goal is to create a large, high-resolution mosaic that may used to quickly inspect the entire scene shot by a camera making a single pass underneath the vehicle. The generated mosaics provide efficient and complete representations of video sequences. Several constraints are placed on the video data in order to facilitate the assumption that the entire scene in the sequence exists on a single plane. Thus, a single mosaic is used to represent a single video sequence. Motion analysis is based on phase correlation in this case.

\section{TIME WARPING FOR IMAGE-BASED RENDERING}

\section{S. Peleg (Israel)}

2D image alignment methods are applied successfully for mosaicing aerial images, but fail when the camera moves in a 3D scene. Such methods can not handle 3D parallax, resulting in distorted mosaicing. General egomotion methods are slow, and do not have the robustness of $2 \mathrm{D}$ alignment methods.

We propose to use the $\mathrm{x}$-y-t space-time volume as a tool for depth invariant mosaicing. When the camera moves on a straight line in the " $\mathrm{x}$ " direction, a " $\mathrm{y}$ - $\mathrm{t}$ " slice of the spacetime volume is a panoramic mosaic, while a "x-t" slice is an EPI plane. Time warping, which is a resampling of the "t" axis, is used to form straight feature lines in the EPI planes. This process will simultaneously give best panoramic mosaic in the "y-t" slices.

This approach is as robust as 2D alignment methods, while giving depth invariant motion (ego motion). Extensions for two dimensional camera motion on a plane are also described, with applications for 2D mosaicing, and for image based rendering such as light field.

\section{GENERATING MOSAICS WITH MINIMUM DISTORTIONS}

\section{Feldman (Israel)}

Manifold mosaicing is a fast and robust way to summarize video sequences captured by a translating camera. It is also useful for rendering compelling 3D visualizations from a video without estimating the 3D structure of the scene. However, since the result mosaics are not perspective images, their geometry is inherently distorted. These mosaics are commonly referred to as multi-perspective images, or multi-perspective mosaics.

In this paper we consider the following question: Given a video captured by a moving camera, what is the best multi-perspective mosaic that can be generated from it? What is the mosaic with the best combination of large field-of-view and minimal geometric distortions? We define the necessary conditions for a good mosaic and a quantitative criterion for the geometric distortions, and derive analytically the optimal mosaic under this criterion. Results on video sequences confirm that indeed the optimal mosaic has significantly better quality than those generated by other techniques.

\section{IMAGE BASED RENDERING USING OMNIDIRECTIONAL X-SLITS IMAGES}

\section{H. Bakstein (Czech Republic)}

We focus on application of omnidirectional sensors in image based rendering (IBR). We use a camera equipped with a circular fisheye lens with a field of view of 180 degrees. By rotating the camera on a circular path, we capture an image sequence and organize the images in a so called 'omnidirectional image volume'. We show that different cuts through this volume can lead to several instances of non-central cameras, namely concentric mosaics and X-slits images. Then, we focus on X-slits image based rendering. We discuss two issues. First, we analyze the number of images required to represent a scene. We want to minimize this number while preserving high visual fidelity of the IBR rendered images. Second, we study distortions inherent to the X-slits method, which displays object at different scene depth with different aspect ratios. We show that most of the distortion is not observed by people, as long as a perceptually dominant object is undistorted. 


\title{
Part IV - NAVIGATION
}

\author{
EXPLOITING PANORAMIC VISION \\ FOR ANGLE-BASED ROBOT HOMING
}

\author{
K. Bekris (USA), A. Argyros (Greece), and L. Kavraki (USA)
}

Omni-directional vision allows for the development of techniques for mobile robot navigation that have minimum perceptual requirements. In this work, we focus on robot navigation algorithms that do not require range information or metric maps of the environment. More specifically, we present a homing strategy that enables a robot to return to its home position after executing a long path. The proposed strategy relies on measuring the angle between pairs of features extracted from panoramic images, which can be achieved accurately and robustly. In the heart of the proposed homing strategy lies a novel, local control law that enables a robot to reach any position on the plane by exploiting the bearings of at least three landmarks of unknown position, without making assumptions regarding the robot's orientation and without making use of a compass. This control law is the result of the unification of two other local control laws which guide the robot by monitoring the bearing of landmarks and which are able to reach complementary sets of goal positions on the plane. Long-range homing is then realized through the systematic application of the unified control law between automatically extracted milestone positions connecting the robot's current position to the home position. Experimental results, conducted both in a simulated environment and on a robotic platform equipped with a panoramic camera validate the employed local control laws as well as the overall homing strategy. Moreover, they show that panoramic vision can assist in simplifying the perceptual processes required to support robust and accurate homing behaviors.

\section{NAVIGATION AND GRAVITATION}

\author{
S.S. Beauchemin, M.T. Kotb, and H.O. Hamshari (Canada)
}

We propose a mathematical model for vision-based autonomous navigation on general terrains. Our model, a generalization of Mallot's inverse perspective, assumes the direction of gravity and the speed of the mobile agent to be known, thus combining visual, inertial, and navigational information into a coherent scheme. Experiments showing the viability of this approach are presented and a sensitivity analysis with random, zero-mean Gaussian noise is provided.

\section{OMNIDIRECTIONAL VISION FOR SOCCER PLAYING ROBOTS}

\section{S. Behnke (Germany)}

The talk presents an omnidirectional vision system, which has been developed for the RoboCup MidSize league. The tasks of this system are to perceive the key objects ball, goals, corner poles, and other players, and to localize the robot on the soccer field. This needs to be done in real time, with limited computing power, imprecise optics, vibrations, variations in lighting, and so on. To solve the task despite these difficulties, a model of the environment is used. The color-coding of the objects makes reliable object-detection possible. The input images are captured in YUV color space. Pixels are classified by color to create low-resolution single-color images. The most significant blobs of each color are detected. Their coordinates are estimated in an egocentric frame. The yellow and blue color images are transformed to an unwarped image. Here, corner poles and goals are detected and verified. Based on these observations, the robot pose is estimated. The estimated pose is used to transform ball and obstacle coordinates into allocentric coordinates. The system makes use of confidences and predictions to track the objects of interest. It utilizes various heuristics to exclude common error sources. The system was implemented on a subnotebook onboard the FU-Fighters MidSize robots. It runs at 30fps, parallel to behavior control and communication. The described omnidirectional vision system performed well at the RoboCup competition in Fukuoka, Japan. 


\title{
Part V - SENSORS AND OTHER MODALITIES
}

\section{BEYOND TRICHROMATIC IMAGING}

\author{
E. Angelopoulou (USA)
}

An integral part of computer vision and graphics is modeling how a surface reflects light. There is a substantial body of work on models describing surface reflectance ranging from purely diffuse to purely specular. One of the advantages of diffuse reflectance is that the color and the intensity of the reflected light are separable. For diffuse materials, the objective color of the surface depends on the chromophores present in the material and is described by its albedo. We will show that for diffuse reflectance, multispectral image analysis allows us to isolate the albedo of a surface. By computing the spectral gradients, i.e. evaluating the partial derivatives with respect to wavelength at distinct wavelengths, one can extract a quantity that is independent of the incident light and the scene geometry. The extracted measurement is the partial derivative of the albedo with respect to wavelength.

In specular highlights the color and the intensity of a specularity depend on both the geometry and the index of refraction of the material, which in turn is a function of wavelength. Though the vision and graphics communities often assume that for non-conductive materials the color of the specularity is the color of the light source, we will show that under multispectral imaging this assumption is often violated. Multispectral image analysis supports the underlying theory which predicts that even for non-metallic surfaces the reflectivity ratio at specularities varies with both wavelength and angle of incidence. Furthermore, the spectral gradients of specular highlights isolate the Fresnel term up to an additive constant.

\section{D OPTICAL FLOW IN GATED MRI CARDIAC DATASETS}

\section{J. Barron (Canada)}

We extend the 2D "strawman" least squares and regularization approaches of Lucas and Kanade and Horn and Schunck and show flow fields (as XY and XZ 2D flows) for a beating heart. The flow not only can captures the expansion and contraction of the various parts of the heart motion but also can capture the twisting motion of the heart while it is beating.

\section{IMAGING THROUGH TIME}

\author{
R. Pless (USA)
}

Many classical vision algorithms mimic the structure and function of the human visual system — which has been an effective tool for driving research into stereo and structure from motion based algorithms. However, for problems such as surveillance, tracking, anomaly detection and scene segmentation; problems that depend significantly on local context, the lessons of the human visual system are less clear. For these problems, significant advantages are possible in a "persistent vision" paradigm that advocates collecting statistical representations of scene variation from a single viewpoint over very long time periods. This chapter motivates this approach by providing a collection of examples where very simple statistics, which can be easily kept over very long time periods, dramatically simplify scene interpretation problems including segmentation and feature attribution.

\section{UBIQUITOUS AND WEARABLE COMPUTER SYSTEMS}

\section{T. Matsuyama (Japan)}

Capturing multi-view images by a group of spatially distributed cameras is one of the most useful and practical methods to extend utilities and overcome limita- tions of a standard pinhole camera: limited size of visual field and degeneration of 3D information. This paper gives an overview of our research activities on multi-view image 
analysis. First we address a ubiquitous vision system, where a group of network-connected active cameras are embedded in the real world to realize 1) wide-area dynamic 3D scene understanding and 2) versatile 3D scene visualization. To demonstrate utilities of the system, we developed a coopera- tive distributed active object tracking system and a 3D video generation system. The latter half of the paper discusses a wearable vision system, where multiple active cameras are placed nearby around human eyes to share the visual field. To demonstrate utilities of the system, we developed systems for 1) real time accurate estimation of 3D human gaze point, 2) 3D digitization of a hand-held object, and 3) estimation of 3D human motion trajectory.

\title{
COMPUTER ASSISTED DIAGNOSIS (CAD) FOR THE RAPID AUTOMATED MEASUREMENT OF BODY FAT TISSUE FROM WHOLE BODY MRI
}

\author{
P. F. Whelan (Ireland)
}

This talk will examine the technical issues relating to the feasibility of using Computer Assisted Diagnosis (CAD) techniques to automatically identify, localize, and accurately measure body fat tissue from a rapid whole body MRI exam. The aim of this work is the provision of an automated system, which assesses subjects whole body MRI scans and which provides numerical and visual feedback to illustrate the findings. The system generates real time results allowing for an initial assessment to be performed immediately following the completion of an MRI scan. The paper will focus on the specific issues relating to the formation, volume reconstruction, image processing and analysis of the whole body images. A working system and details of a prospective investigative study of 42 volunteers will be presented. 